\title{
EDITORIAL
}

\section{Efficiency and effectiveness}

Not only the United Kingdom is becoming increasingly aware of politicians' desires to control expenditure on health care. Both the epitomy of private medicine-the USand the pioneer of socialized medicine-the UK-are aware of inefficiency in the service and are seeking methods to correct it. We are often led to believe that the National Health Service is grossly inefficient, yet the health care we provide costs us about half what it costs the Americans (Fry et al., 1984). This is not a plea for complacency, merely perspective. Whenever efficiency is discussed clinicians usually feel the real subject under discussion is a reduction in expenditure with a corresponding reduction in health care. This is not always the case, but it is often so. However, there are areas of practice where efficiency can be improved and effectiveness increased at the same time. Accident and emergency departments hold dear many ancient customs and practices. Many are tried and tested but some are just tried. Emergency medicine has for too long been the province of junior and inexperienced doctors working short-term contracts without adequate senior supervision. These fledgling doctors learn to survive in this hostile environment by falling back on 'traditional' treatments. Certainly, they feel more confident to survive litigation if they have used such traditional methods. However, now that emergency medicine is being recognized as a specialty and senior doctors are engaged in first contact with emergency patients, we have opportunities to evaluate all our treatment methods and to audit and analyse their efficiency continually. For example, the treatment of soft tissue abscesses has long been the province of emergency departments and incision and drainage has become a routine procedure. A pioneer specialist in accident and emergency medicine in the UK advocated some time ago that this time-honoured procedure might be questionable. Ellis (1953) advised the administration of parenteral antibiotics prior to incision of an abscess, and then primary closure with sutures. What seemed like heresy at the time has produced no serious complications in the hands of people who have used this technique but has never gained widespread acceptance. Things may now change as it has been shown that superficial abscesses can be incised, drained and closed with sutures without even the benefit of antibiotic cover (Stewart et al., 1985). Clearly, more studies need to be undertaken, but it may be that the time-honoured practice of incision and drainage of abscesses followed by repeated packs involving costly visits to the hospital or home visits by the district nurse may become a thing of the past for many patients. It must be stressed that a prerequisite of primary closure is adequate curettage. This means adequate anaesthesia must be employed and this usually means a general anaesthetic. Transient bacteraemia always occurs, but this is only harmful to patients who have prostheses which may become infected, e.g. prosthetic heart valve or hip replacement. Such patients will require antibiotic cover.

In most casualty departments if you sit still long enough, even if only as a visitor, you 


\section{Editorial}

will be given a tetanus toxid booster! When horse serum was used great care and $\frac{\mathbb{Q}}{\varrho}$ reticence was employed in the administration of immunization against tetanus because $\frac{3}{\infty}$ of the frequency of serious and sometimes fatal reactions. With the advent of tetanus? toxoid and the extremely low incidence of any side-effects, subsequent euphoria $\overrightarrow{\vec{F}}$ resulted in lowering of the threshold for giving tetanus toxoid. This may be no bado thing. The vaccine is cheap and the consequences of tetanus are extremely serious. $\frac{\bar{E}}{\bar{n}}$ Fortunately, tetanus is rare in the UK at 30-50 cases per year (Public Health $\frac{\bar{c}}{\bar{\phi}}$ Laboratory Service, 1985) and most cases occur in patients not previously immunized $\varrho$ and not seeking or getting appropriate surgical treatment for an 'at risk' wound. Recent ${ }^{\mathscr{N}}$ evidence from Canada (Mathias \& Schediter, 1985) shows that if primary vaccination is. good then immunity may last a lot longer than previously thought. These authors even $\vec{\omega}$ question the routine giving of tetanus toxoid booster to a tetanus prone wound in $\frac{D}{\Phi}$ patients who have previously had a full course. The difficulty for people in an 3 . emergency department is that patients are not usually aware of what vaccination they? have had and it may prove impossible to establish the immune status of an individual. $\stackrel{\omega}{\vec{\theta}}$ What this recent evidence can tell us for now, however, is that we must make greater $\varnothing$ efforts to ensure that everybody has a full course of primary vaccination and once this is $\frac{}{5}$ ensured prevention of tetanus will rely more on surgical debridement of the wound than $\vec{\oplus}$ the giving of a tetanus toxoid booster. When the patient has clearly not been given a $\mathbb{D}$ course of immunization then passive immunization with immunoglobulin is indicated, $\mathbb{D}$ as highlighted by the case reported in a previous issue of this journal (Young, 1985).

Many X-ray examinations are taken in an emergency department for clinical curiosity rather than clinical necessity. For example, an X-ray of a little toe may illuminate fracture, but it will not shed light on the (limited) choice of treatment. Plain radiograp of the abdomen are often ordered, but are they really helpful? Two studies have recent度 looked at the value of the plain abdominal radiograph in diagnosing ureteric calculi, but have shown it to be of little help (Roth et al., 1985; Zaugerle et al., 1985). Even when X-

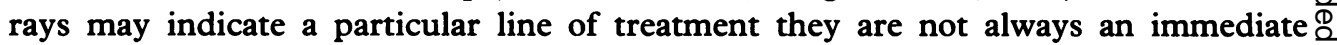
necessity. The cost of out-of-hours X-rays in British practice is a great burden to the $\vec{O}$ NHS. Certain X-rays, e.g. of the skull, will always need to be taken no matter what the 3 time of day as they have a direct influence on patient management, i.e. a normal skull Xray is one of the indications for sending a head-injured patient home. However, if for example an ankle is not clinically in need of immediate reduction and there is no 3 . neurovascular impairment, then X-ray may not be indicated in the middle of the night. $\frac{\sigma}{3}$ The patient can be made comfortable with a supportive bandage and non-weight bearing on crutches, and given adequate analgesia overnight. $\mathrm{X}$-ray next morning will not introduce any serious delay in treatment and may be extremely cost-effective.

Giving a general anaesthetic to an elderly patient at night may be the start rather than the end of his troubles. The patient often needs admission to a bed overnight from whence they may find difficulty in escaping. Many general anaesthetics are given for $N$ injuries to the forearm which could often be managed by local anaesthetic techniques. Wardrope et al. (1985) have shown that even local infiltration of a fracture haematoma $\mathrm{N}$ in the forearm does not produce any serious problems, although a Bier's block $\sigma$ technique is clearly more effective. The cost savings to be accrued from this technique may be considerable in both financial and personal terms, but it is important that the guidelines recommended by the Casualty Surgeons Association (CSA) are adhered to at ? all times (Casualty Surgeons Association, 1983). 
Many thousands of patients are admitted for overnight observation following minor head injury. Great care has to be taken in any attempt to reduce this number as the penalty for sending home a missed intracranial haematoma may be death. Timehonoured criteria for the admission of patients for overnight observation have always included a history of unconsciousness or amnesia. This has meant a large number of healthy patients spending short but expensive times in hospital beds. Gorman in this issue demonstrates that these two criteria are not absolute indications for hospital admission following head injury, and the reduction in unnecessary admissions may be greater than $30 \%$. Similar findings have been reported by Miller \& Jones (1985).

A recent document from the Department of Health and Social Security for the management of deliberate self-harm recommends that suitably trained medical practitioners other than psychiatrists may undertake the psychosocial assessment of patients who have deliberately harmed themselves (DHSS, 1984). Casualty officers can be trained in the assessment of suicide risk alongside their psychiatric colleagues and so improve the efficiency with which these patients are dealt with and hopefully improve the effectiveness of their treatment.

These are some examples where increases in efficiency can be matched by increases in effectiveness. Particularly in the case of head injuries, medical and nursing staff spend the majority of their time dealing with healthy people who make uneventful recoveries; this is not only inefficient but unlikely to be effective in spotting the occasional patient who develops complications. A reduction in the course of antibiotics for likely soft tissue infection from 7 days to 5 days may accrue an enormous saving. Similarly, it has been shown that Paracetamol is as good an analgesic for accident and emergency departments as any and is a lot cheaper (Yates et al., 1984). All aspects of accident and emergency care should be analysed both for efficiency but more importantly for effectiveness.

\section{Casualty Surgeons Association}

It is with great pleasure that I am able to announce that the Casualty Surgeons Association of the United Kingdom, at its annual general meeting in Birmingham in April this year, formally adopted Archives of Emergency Medicine as its official journal. Membership of the association will now include subscription to the journal. It is a most welcome development, and I hope the relationship will be long and fruitful.

\section{A. D. REDMOND}

Editor

\section{REFERENCES}

Casualty Surgeons Association (1983) Bier's Blocks (Intravenous Regional Anaesthesia). Code of Practice. U.K., Casualty Surgeons Association.

Department of Health and Social Security (1984) Health Notice (84)25. London, Her Majesty's Stationery Office.

Ellis M. (1953) The new treatment of ischiorectal abscess. University of Leeds Medical fournal 2, 84-6.

Fry J., Brooks D. \& McColl I. (1984) NHS Data Book. Lancaster, MTP Press. 


\section{Editorial}

Mathias R. G. \& Schediter M. T. (1985) Booster immunization for diphtheria and tetanus: no evidence of $\frac{\mathbb{Q}}{0}$ need in adults. Lancet i, 1089-90.

Miller J. D. \& Jones P. A. (1985) The work of a regional head injury service. Lancet i, 1141-3.

Public Health Laboratory Service (1985) Communicable disease surveillance centre: tetanus surveillance: England and Wales 1981-3. British Medical fournal 290, 289-90.

Roth C. S., Bowyer B. A. \& Berquist T. H. (1985) Utility of the plain abdominal radiograph for disguising

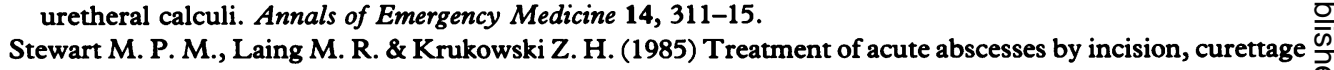
and primary suture without antibiotics: a controlled clinical trial. British fournal of Surgery 72, 66-7.

Wardrope J., Flowers M. \& Wilson D. H. (1985) Comparison of local anaesthetic techniques in the reduction $\cong$ of Colles' fracture. Archives of Emergency Medicine 2, 67-72.

Yates D. W., Laing G. S., Peters K. \& Kumar K. (1984) Mild analgesics and the accident and emergency $\vec{\circ}$ department: cost and safety more important than potency? Archives of Emergency Medicine 1, 197-204.

Young T. B. (1985) An indication for human tetanus immunoglobin in unimmunized patients. Archives of

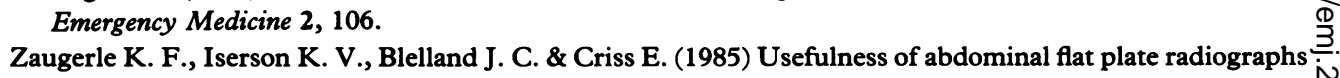
in patients with suspected uretheral calculi. 